\title{
LncRNA HOTAIR Regulates CCND1 and CCND2 Expression by Sponging miR-206 in Ovarian Cancer
}

\author{
Lei Chang Ruixia Guo Zhongfu Yuan Huirong Shi Dongya Zhang \\ Department of Gynecology, the First Affiliated Hospital of Zhengzhou University, Zhengzhou, China
}

\author{
Key Words \\ LncRNA HOTAIR • miR-206 • CCND1 • CCND2 • Ovarian cancer
}

\begin{abstract}
Background/Aims: The long noncoding RNA homeobox (HOX) transcript antisense intergenic RNA (HOTAIR) has been demonstrated to be a vital modulator in the proliferation and metastasis of ovarian cancer cells, but its potential molecular mechanism remains to be elucidated. In the current study, we aimed to uncover the biological role of IncRNA HOTAIR and its underlying regulatory mechanism in the progression and metastasis of ovarian cancer. Methods: HOTAIR expression was detected by quantitative RT-PCR (qRT-PCR) and northern blotting. The SKOV3 ovarian cancer cell line was chosen for the subsequent assays. In addition, the molecular mRNA and protein expression levels were examined by qRT-PCR and western blotting. The competitive endogenous RNA (ceRNA) mechanism was validated by bioinformatics analysis and a dual luciferase reporter gene assay. Results: HOTAIR expression was significantly higher in ovarian carcinoma tissues and cell lines than in the control counterparts. Both CCND1 and CCND2 were downstream targets of miR-206. The inhibition of HOTAIR elevated the expression of miR-206 and inhibited the expression of CCND1 and CCND2. Moreover, CCND1 and CCND2 were highly expressed in ovarian cancer tissues, and their expression was positively correlated with HOTAIR expression. Finally, the functional assays indicated that the anticancer effects of miR-206 could be rescued by the simultaneous overexpression of either CCND1 or CCND2 in ovarian cancer. Conclusion: HOTAIR enhanced CCND1 and CCND2 expression by negatively modulating miR-206 expression and stimulating the proliferation, cell cycle progression, migration and invasion of ovarian cancer cells.
\end{abstract}

\section{Introduction}

Ovarian cancer is one of the three most prevalent gynecological malignant tumors and has the highest mortality rate among all gynecological cancers worldwide [1, 2]. Despite the advancements in surgical, chemotherapeutic and radiotherapeutic treatment, the 
prognosis of OC remains unsatisfactory, with an overall 5-year survival rate of approximately $30 \%[3,4]$. Additionally, patients often experience an extremely poor outcome because of the limitations of the therapeutic strategies, rapid progression of the disease and frequent occurrence of cancer metastasis and recurrence. Hence, it is urgent to understand the underlying molecular mechanisms of OC progression to identify novel targets for OC therapy.

In light of the results of recent genome studies, the importance of the non-protein-coding portion of the genome in carcinogenesis and metastasis has been increasingly recognized [5, 6]. Among non-protein-coding segments, miRNAs (20 - $25 \mathrm{nt}$ in length) have been proven to regulate gene expression in fundamental biological processes and the pathogenesis of human cancers $[7,8]$. In addition, it is noteworthy that miRNAs are functional molecules in many cancers [9]. In addition, the long noncoding RNA (lncRNA) family is a heterogenous family that is represented by long intergenic RNAs (defined by position), circular RNAs (circRNAs, defined by structure), competing endogenous RNAs (ceRNAs, defined by functions), etc. One of the important functions of IncRNAs is serving as ceRNAs to sponge miRNAs, which in turn regulates relevant miRNA targets [10].

Homeobox (HOX) transcript antisense intergenic RNA (HOTAIR), the transcript from the HOXC locus, has been demonstrated to be a vital modulator in the deterioration of different cancers $[11,12]$. Many studies have suggested that human IncRNA HOTAIR functioned as a ceRNA in a series of tumors and was positively correlated with malignant processes and poor outcomes [13-16]. Recent studies also illustrated that HOTAIR was aberrantly highly expressed in ovarian carcinoma and was linked to the proliferation and metastasis of ovarian cancer cells [17]. Nevertheless, its biological role and potential molecular mechanism in ovarian carcinoma remain to be elucidated.

CCND1 and CCND2, whose upregulation was associated with more advanced tumors and tumor metastasis, are common cancer promoters in humans $[18,19]$. For instance, CCND1 downregulation could suppress proliferation and cell cycle progression in hepatocellular carcinoma cells [20]. Activation of CCND2 could promote the development and progression of thyroid cancer [21], and CCND1, which is highly expressed in epithelial ovarian cancer cell lines, could promote cell proliferation and inhibit apoptosis [22]. However, the functions of CCND1 and CCND2 in ovarian carcinoma still need to be further elucidated.

In the present study, we investigated the role of HOTAIR, miRNA, CCND1 and CCND2 in ovarian cancer as well as the correlation between these players. Our study could shed new light on the understanding of ovarian carcinogenesis and the development of novel drug targets for ovarian cancer therapy.

\section{Materials and Methods}

\section{Sample collection and culture}

A total of 92 pairs of ovarian carcinoma and corresponding paracarcinoma tissues were randomly obtained from Chinese patients who had undergone surgery at the First Affiliated Hospital of Zhengzhou University between 2014 and 2016. All cases were carefully diagnosed as ovarian cancer on the basis of histopathological evaluation, and none of the experimental subjects received any other adjuvant therapies prior to surgery. Then, samples were collected, frozen and stored at $-80^{\circ} \mathrm{C}$. Informed consent was obtained from each participant. This study was approved and supervised by the First Affiliated Hospital of Zhengzhou University, and the relevant clinicopathological features of the patients are listed in Table 1.

Four ovarian carcinoma cell lines (COV362, SKOV3, OVCAR3 and A2780) and one human ovarian epithelial cell line (HOSEpiC) were obtained from the Institute of Biochemistry and Cell Biology of the Chinese Academy of Sciences (Shanghai, China) and were cultured in RPMI-1640 medium supplemented with $10 \%$ fetal bovine serum (FBS, GIBCO, Thermo Fisher Scientific, Inc., Waltham, MA, USA), $100 \mathrm{U} / \mathrm{ml}$ penicillin and $100 \mathrm{mg} / \mathrm{ml}$ streptomycin in a humidified atmosphere of $5 \% \mathrm{CO}_{2}$ at $37{ }^{\circ} \mathrm{C}$. 
Table 1. Correlation of the expression of HOTAIR, CCND1 and CCND2 with clinicopathological features. Chisquared test $\left({ }^{*} \mathrm{P}<0.05\right)$

\begin{tabular}{|c|c|c|c|c|c|c|c|c|c|c|}
\hline \multirow{2}{*}{ Clinicopathological parameters } & \multirow{2}{*}{ Number } & \multicolumn{3}{|c|}{ HOTAIR expression } & \multicolumn{3}{|c|}{ CCND1 expression } & \multicolumn{3}{|c|}{ CCND2 expression } \\
\hline & & Low & High & $\mathrm{P}$ & Low & High & $\mathrm{P}$ & Low & High & $\mathrm{P}$ \\
\hline \multicolumn{11}{|l|}{ Age (years) } \\
\hline$\leq 55$ & 41 & 24 & 17 & \multirow{2}{*}{0.1420} & 20 & 21 & \multirow{2}{*}{0.8339} & 18 & 23 & \multirow{2}{*}{0.2943} \\
\hline$>55$ & 51 & 22 & 29 & & 26 & 25 & & 28 & 23 & \\
\hline \multicolumn{11}{|l|}{ Histological grade } \\
\hline Well & 27 & 19 & 8 & \multirow{3}{*}{0.0094} & 18 & 9 & \multirow{3}{*}{0.1177} & 16 & 11 & \multirow{3}{*}{0.1429} \\
\hline Moderate & 48 & 23 & 25 & & 21 & 27 & & 25 & 23 & \\
\hline Poor & 17 & 4 & 13 & & 7 & 10 & & 5 & 12 & \\
\hline \multicolumn{11}{|l|}{ FIGO stage } \\
\hline $\mathrm{I}+\mathrm{II}$ & 60 & 35 & 25 & \multirow{2}{*}{0.0286} & 33 & 27 & \multirow{2}{*}{0.1891} & 38 & 22 & \multirow{2}{*}{0.0005} \\
\hline III+IV & 32 & 11 & 21 & & 13 & 19 & & 8 & 24 & \\
\hline \multicolumn{11}{|l|}{ Metastasis } \\
\hline No & 45 & 28 & 17 & \multirow{2}{*}{0.0218} & 21 & 24 & \multirow{2}{*}{0.5315} & 26 & 19 & \multirow{2}{*}{0.1443} \\
\hline Yes & 47 & 18 & 29 & & 25 & 22 & & 20 & 27 & \\
\hline
\end{tabular}

\section{qRT-PCR}

Total RNA was isolated from tissues and cells using TRIzol reagent (Invitrogen, Carlsbad, CA, USA) and the mirVana ${ }^{\mathrm{TM}}$ miRNA Isolation Kit (Thermo Fisher Scientific) [23]. For mRNA and IncRNA, RNA was reversely transcribed into cDNA using the M-MLV Reverse Transcription Kit (Invitrogen, Carlsbad, CA, USA). The thermal cycling profile was as follows: denaturation for 30 s at $95^{\circ} \mathrm{C}$, annealing for $45 \mathrm{~s}$ at $56^{\circ} \mathrm{C}$, and extension for $45 \mathrm{~s}$ at $72{ }^{\circ} \mathrm{C}$. Each PCR reaction was carried out for 35 cycles using the ABI 7500 Real-Time PCR system (Applied Biosystems, Foster City, CA, USA). RT-PCR analysis was performed with the SYBR Premix Ex Taq ${ }^{\mathrm{TM}}$ Kit (TaKaRa, Dalian, China). For the detection of microRNA expression, reverse transcription was performed following the Applied Biosystems TaqMan MicroRNA Assay protocol (Cat. \# 442797, Applied Biosystems, Carlsbad, CA, USA). MiRNA expression assays were performed in triplicate using the SYBR PrimeScriptTM miRNA RT-PCR Kit (TaKaRa Biotech). The primer sequences are presented in Table 2. All the target and reference sequences were amplified in triplicate. The results were normalized to the expression of GAPDH (for IncRNAs and mRNA) or U6 (for miRNAs), and the relative expression levels of the targets were calculated using the $2^{-\Delta \Delta C t}$ method.

\section{Northern blot analysis}

For all the groups, $20 \mu \mathrm{g}$ of total RNA was analyzed on a $12 \%$ denaturing 12 polyacrylamide-urea (7.5 M) gel and was transferred onto a Hybond- $\mathrm{N}^{+}$nylon membrane (Amersham, Freiburg, Germany). The blotted RNA was crosslinked to the membranes under ultraviolet light for $30 \mathrm{~s}$ at $1,200 \mathrm{mJoule} / \mathrm{cm}^{2}$. The antisense Starfire probe was incubated with the crosslinked membranes at $37{ }^{\circ} \mathrm{C}$ in a shaking oven. The probes were decanted and washed for $15 \mathrm{~min}$. Then, the membranes were exposed for 20-40 h to Kodak XAR- 5 films (Eastman Kodak, Rochester, NY, USA). The human U6 snRNA probe was used as a control; its sequence was 5'-GCAGGGGCCATGCTAATCTTCTCTGTATCG-3'. The exposure time for the U6 control probe varied between 15 and 30 min.

\section{Western blot}

Cells were lysed using radioimmunoprecipitation assay (RIPA) buffer with protein extraction reagent (Beyotime, Shanghai, China) supplemented with phenylmethanesulfonyl fluoride (PMSF) (Roche, Basel, 


\section{Cellular Physiology Cell Physiol Biochem 2018;49:1289-1303 and Biochemistry Published \begin{tabular}{l|l} 
DOI: 10.1159/000493408 & $\begin{array}{l}\text { C } 2018 \text { The Author(s). Published by S. Karger AG, Basel } \\
\text { www.karger.com/cpb }\end{array}$
\end{tabular} \\ Chang et al.: Functions and Related Mechanism of HOTAIR in OC}

Switzerland). Approximately $25 \mu \mathrm{g}$ of protein was separated by $10 \%$ sodium dodecyl sulfate polyacrylamide gel electrophoresis (SDS-PAGE), transferred onto nitrocellulose membranes (Sigma, St. Louis, Mo, USA), and detected with primary antibodies against CCND1 CCND2, or GAPDH (Abcam, Cambridge, UK). The membranes were incubated at $4{ }^{\circ} \mathrm{C}$ overnight, incubated with AP-conjugated secondary antibodies and detected by enhanced chemiluminescence (ECL) reagents.

\section{Cell transfection}

The small interfering RNA (siRNA) against HOTAIR (siHOTAIR), the scramble negative control siRNA (NC), miR-206 mimic, mimic control, miR-206 inhibitor, inhibitor control, Lenti-vpak packaging systemCCND1, Lenti-vpak packaging system-CCND2 and Lenti-vpak packaging system-oligo were synthesized by GenePharma (Shanghai, China). The Lenti-vpak packaging system-oligo, mimic control and inhibitor control servedasnegativecontrols. Therelevantsequenceswereasfollows: siHOTAIR:5'-GCAACCTAAACCAGCAATT-3'; siRNA-control: 5'-GCATCAACAACCGAACATT-3'; miR-206 mimic: F: 5'-UGGAAUGUAAGGAAGUGUGUGG-3' and R: 5'-ACACACUUCCUUACAUUCCAUU-3'; mimic control: F: 5'-UUCUCCGAACGUGUCACGUTT-3' and R: 5'-ACGUGACACGUUCGGAGAATT-3'; miR-206 inhibitor: 5'-CCACACACUUCCUUACAUUCCA-3'; and inhibitor control: 5'-CAGUACUUUUGUGUAGUACAA-3'. Cells in the logarithmic growth phase were harvested $24 \mathrm{~h}$ before transfection. The cells were digested in trypsin and maintained in medium for resuspension. The cells were plated in 6-well plates at a density of $1 \times 10^{6}$ cells per well and cultured in an incubator for $24 \mathrm{~h}$ at 37 ${ }^{\circ} \mathrm{C}$ in $5 \% \mathrm{CO}_{2}$ until the confluence reached $80-90 \%$. The transfections were performed with Lipofectamine 2000 (Invitrogen, Carlsbad, USA) following the manufacturer's instructions. The medium was replaced with complete medium $6 \mathrm{~h}$ after transfection.

\section{MTT assay}

SKOV3 cells were seeded in a 96-well plate $\left(2 \times 10^{3}\right.$ cells/well) and incubated overnight $\left(37{ }^{\circ} \mathrm{C}, 5 \%\right.$ $\mathrm{CO}_{2}$ ). Twenty microliters of MTT solution (Sigma, St. Louis, Mo, USA) was added to each well at $5 \mathrm{mg} / \mathrm{ml}$ in PBS, and the plates were centrifuged at $150 \mathrm{rpm}$ for $5 \mathrm{~min}$. The cells were cultured for another $5 \mathrm{~h}$, after which the medium was removed. Then, the formazan was resuspended in $200 \mu \mathrm{l}$ of DMSO and the plates were centrifuged at $150 \mathrm{rpm}$ for $5 \mathrm{~min}$. The optical density at $450 \mathrm{~nm}$ was measured using an ELISA reader (Model 680; Bio-Rad, Hercules, CA, USA).

\section{Colony formation assay}

After transfection, the medium was removed, and the cells were rinsed with $10 \mathrm{ml}$ of PBS. Four milliliters of $0.25 \%$ trypsin was added to the cells, which were incubated at $37{ }^{\circ} \mathrm{C}$ for $2-5$ min until they appeared round. The cells were then resuspended in $10 \mathrm{ml}$ of medium supplemented with $10 \%$ FBS. The cell number was counted using a hemocytometer. A total of 500 cells was seeded into each 6 -cm dish. After continuous culture for approximately 2 weeks until the cells in the control plates formed appropriately sized colonies (a minimum of 50 cells/colony), the medium was discarded and the cells were rinsed with $10 \mathrm{ml}$ of PBS. The cells were fixed with $4 \%$ paraformaldehyde for $5 \mathrm{~min}$ at room temperature. Subsequently, a $0.1 \%$ crystal violet solution was incubated with the cells for $2 \mathrm{~h}$ at room temperature, and the cells were then separated by pipetting in $10 \mathrm{ml}$ of medium supplemented with 10\% FBS. The crystal violet was carefully rinsed off with tap water, and the dishes were air-dried on a table cover at room temperature for up to a few days. Colony numbers were counted using a stereomicroscope (Nikon Eclipse TS100, Tokyo, Japan).

\section{Flow cytometry}

After transfection, cells were harvested, washed in PBS, and fixed in $75 \%$ cold ethanol for $1 \mathrm{~h}$. After being washed twice with PBS, the cells were centrifuged at $850 \mathrm{~g}$, and the supernatant was removed. The cells were treated with $50 \mu \mathrm{l}$ of RNase to ensure that only DNA was stained. The cells were supplemented with $400 \mu \mathrm{L}$ of propidium iodide (PI) $(50 \mu \mathrm{g} / \mathrm{ml})$ and incubated at $37^{\circ} \mathrm{C}$ for $30 \mathrm{~min}$ in the dark in accordance with the Cell Cycle Analysis Kit (Biyuntian, Jiangsu, China) protocol. The fraction of cells in the G0/G1, S and G2/M phases was obtained using a flow cytometer (FACS Calibur, BD Biosciences, San Jose, CA, USA).

Wound healing assay

A wound healing assay was performed to assess cell migration. SKOV3 cells were cultured at a density of $1.0 \times 10^{6}$ cells/well in 6-well plates and grown to a fully confluent monolayer. After $6 \mathrm{~h}$ of serum starvation, 


\section{Cellular Physiology Cell Physiol Biochem 2018;49:1289-1303 \begin{tabular}{ll|l} 
DOI: 10.1159/000493408 & $\begin{array}{l}\text { O 2018 The Author(s). Published by S. Karger AG, Basel } \\
\text { www.karger.com/cpb }\end{array}$ \\
\hline
\end{tabular}}

the cell monolayer was carefully scraped with a sterile pipette tip across the center of each well to create a wound (scratch) and then washed twice with phosphate-buffered saline (PBS) to remove detached cells from the plates. The cells were then cultured in RPMI- 1640 medium supplemented with $0.5 \%$ FBS in a humidified atmosphere of $5 \% \mathrm{CO}_{2}$ at $37{ }^{\circ} \mathrm{C}$. After $24 \mathrm{~h}$, the medium was replaced with PBS, and the wound gap was observed. The wound healing area was evaluated at $0 \mathrm{~h}$ and $24 \mathrm{~h}$ after wounding and was imaged with a microscope. The gap distance of each monolayer was quantitatively evaluated using ImageJ. Five random views of each well were documented, and each experiment was repeated a minimum of three times.

\section{Cell invasion assay}

ECM gel (E1270-5ML, Sigma-Aldrich, St. Louis, MO, USA) was diluted in ice-cold, serum-free DMEM to a final protein concentration of $2 \mathrm{mg} / \mathrm{ml}$ and was then added into the upper compartment of a Transwell insert with an $8.0 \mu \mathrm{m}$ pore size polycarbonate membrane (Corning, Corning, NY, USA). The Transwell plate (lower compartment) and ECM gel were incubated at $37^{\circ} \mathrm{C}$ for $2 \mathrm{~h}$. After PBS washing and trypsin treatment, the cells were washed and resuspended in DMEM at $5 \times 10^{5}$ cells $/ \mathrm{ml}$. The cells $\left(5 \times 10^{5} \mathrm{cell} / \mathrm{ml}\right)$ were supplemented and cultured at $37{ }^{\circ} \mathrm{C}$ for $24 \mathrm{~h}$. After the incubation period, the insert was carefully removed. The cells and ECM gel in the upper compartment of the insert were removed, whereas the cells on the lower side of the insert membrane were fixed with $5 \%$ glutaraldehyde for $10 \mathrm{~min}$ and stained with $1 \%$ crystal violet in $2 \%$ ethanol for $\mathrm{r} 20 \mathrm{~min}$. Excess crystal violet dye was removed by immersing the insert in $\mathrm{ddH}_{2} \mathrm{O}$ for 3-4 s. The insert was dried completely, and the number of cells on the lower side of the membrane was counted under a microscope. A small number of randomly chosen fields was used to obtain the cell count. The average cell number was documented.

\section{Luciferase reporter assay}

For screening the targeting between HOTAIR and miRNA, we cloned the full-length (2370 bp) lncRNA HOTAIR transcript into a pmirGLO dual-luciferase miRNA target expression vector. The recombinant plasmid was then transfected to four groups of SKOV3 cells, which were pretreated with four different kinds of miRNA mimics (miR-92-5p, miR-106b-5p, miR-206 or miR-326 mimics). The oligo control was used as a negative control. For further experiments, a HOTAIR fragment (310 bp) containing the targeted miR206 binding site was amplified and cloned into a pmirGLO dual-luciferase miRNA target expression vector (Promega, Mannheim, Germany) to create the wild-type HOTAIR reporter vector (HOTAIR-Wt). The putative binding site sequence was substituted to produce the mutated HOTAIR vector (HOTAIR-Mut). The sequence of the HOTAIR fragment is as follows:

“TCCTCCTGCTATTAAGATTGCTAGAGAATTGTGTCTTAAACAG
TTCATGAACCCAGAAGAATGCAATTTCAATGTATTTAGTAC
ACACACAGTATGTATATAAACACAACTCACAGAATATATTTTCC
ATACATTGGGTAGGTATGCACTTTGTGTATATATAATAATG
TATTTTCCATGCAGTTTTAAAATGTAGATATATTAATATCTGGA
TGCATTTTCTGTGCACTGGTTTTATATGCCTTATGGAGTAT
ATACTCACATGTAGCTAAATAGACTCAGGACTGCACATTCCTTGT
GTAGGTTGTGTGTGTGTGGTGGTTTTATGCATAAATAAAG
TTTTACATGTGGTGAATATAAA".

The wild-type and mutated vectors for CCND1 and CCND2 were created in a similar fashion. Then, the vectors and the miR-206 mimics or mimic controls were cotransfected into SKOV3 cells, and the dual luciferase reporter assay system (Promega) was used to test luciferase activity.

\section{Statistical analysis and bioinformatics methods}

Statistical analyses were carried out with the SPSS 20.0 software (IBM, SPSS, Chicago, IL, USA). Data were manifested as the mean \pm SD. One-way analysis of variance (ANOVA) or two-tailed Student's t-test was applied to determine the group differences. $P<0.05$ was considered statistically significant. HOTAIR's potential miRNA binding sites were predicted by a computer-aided algorithm from Segal Lab (http://132.77.150.113/pubs/mir07/mir07_prediction.html), RegRNA (http://regrna.mbc.nctu.edu.tw/ $\mathrm{html} /$ prediction.html) and the microRNA.org-target program (www.microRNA.org). 


\section{Results}

HOTAIR was overexpressed in ovarian carcinoma tissues and cell lines

Fig. 1A shows that HOTAIR expression was significantly higher in ovarian carcinoma tissues than it was in the corresponding paracarcinoma tissues. Compared with the HOSEpiC cell line, the ovarian carcinoma cell lines, especially the SKOV3 cell line, showed significantly higher HOTAIR expression (Fig. 1B). Similarly, the northern blot analysis demonstrated that the HOTAIR hybridization signal was stronger in ovarian carcinoma tissues and cell lines than in fresh nontumor specimens and the normal ovarian epithelial cell line (Fig. 1C, 1D).

\section{Inhibition of HOTAIR reduced cell viability, proliferation, migration and invasion}

After transfection with siHOTAIR, HOTAIR expression in the siHOTAIR-treated group was lower than that in the empty vector (Mock) group and the NC group. Expression was detected by qRT-PCR and northern blotting (Fig. 2A). Next, the MTT assay result showed that the downregulation of HOTAIR significantly inhibited SKOV3 cell proliferation in a timedependent manner (Fig. 2B). The number of colonies formed by SKOV3 cells treated with siHOTAIR was significantly less than that of the NC or Mock groups (Fig. 2C), indicating that the downregulation of HOTAIR expression could inhibit ovarian cancer cell proliferation. Furthermore, the depletion of HOTAIR in SKOV3 cells increased the fraction of cells in the G0/G1 phase and decreased the fraction of cells in the $S$ and G2/M phases (Fig. 2D). As shown in Fig. 2E-F, siHOTAIR transfection clearly stimulated SKOV3 cell migration and invasion by approximately $40 \%$.

\section{HOTAIR targeted miR-206, which targeted CCND1 and CCND2}

The luciferase reporter vector scheme is shown in Fig. 3A. The HOTAIR binding sites on miR-92-5p, miR-106b-5p, miR-206 and miR-326 are also shown. The relative luciferase activities in the different groups suggested that HOTAIR significantly suppressed miR-206 expression (Fig. 3B). The miR-373 group was used as the positive control [17]. We confirmed that HOTAIR directly targeted miR-206 (Fig. 3C-D). We detected the expression levels of

Fig. 1. HOTAIR expression was higher in ovarian carcinoma tissues and cell lines. (A) The expression level of HOTAIR was higher in ovarian carcinoma tissues than in the corresponding paracarcinoma tissues. ${ }^{* *} \mathrm{P}<0.01$, compared with the normal tissue group. (B) The expression level of HOTAIR was higher in four ovarian carcinoma cell lines (COV362, SKOV3, OVCAR3 and A2780) than in the human normal ovarian epithelial cell line HOSEpiC. ${ }^{* *} \mathrm{P}<0.01,{ }^{* * *} \mathrm{P}<0.001$,

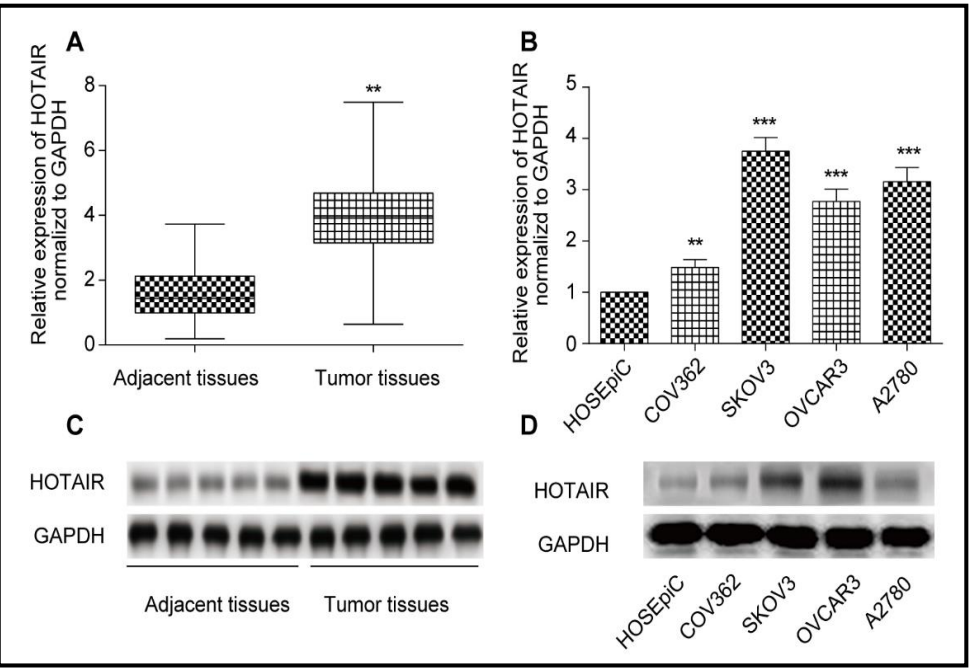
compared with HOSEpiC cells.

(C) The expression level of HOTAIR detected by northern blot was higher in ovarian carcinoma tissues than in paracarcinoma tissues. (D) The expression level of HOTAIR detected by northern blot was higher in the ovarian carcinoma cell lines than in the normal ovarian epithelial cell line. The expression of HOTAIR was normalized to GAPDH mRNA expression. Number of tissues $=92$. 
A

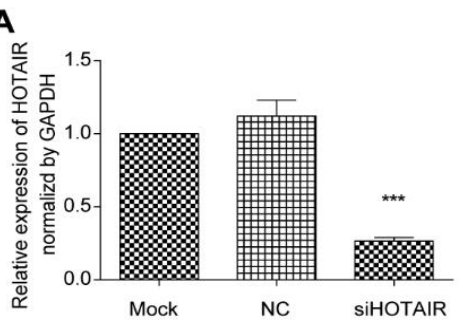

C

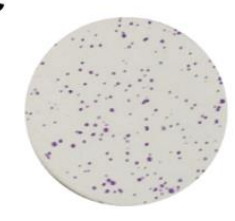

D

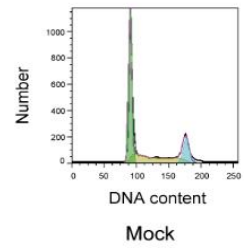

E
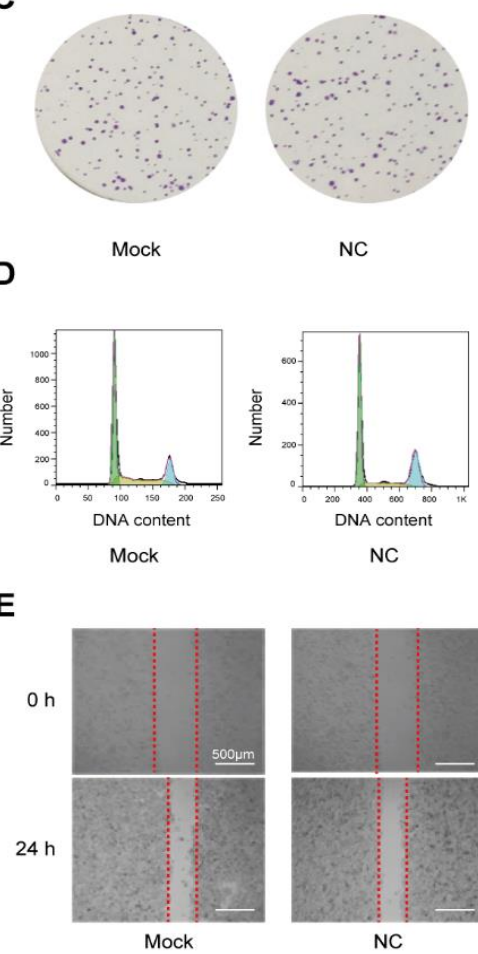

NC

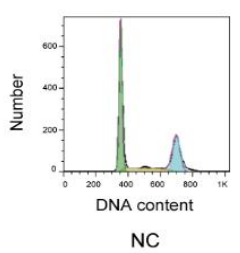

NC

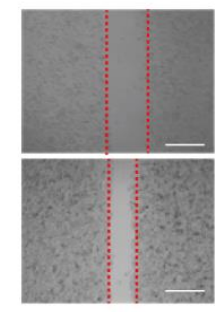

NC

$\mathbf{F}$

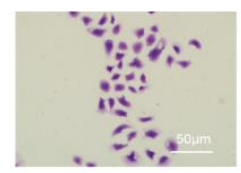

Mock

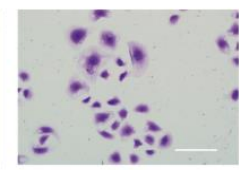

NC

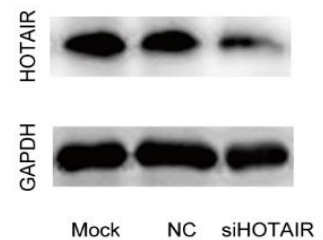

B
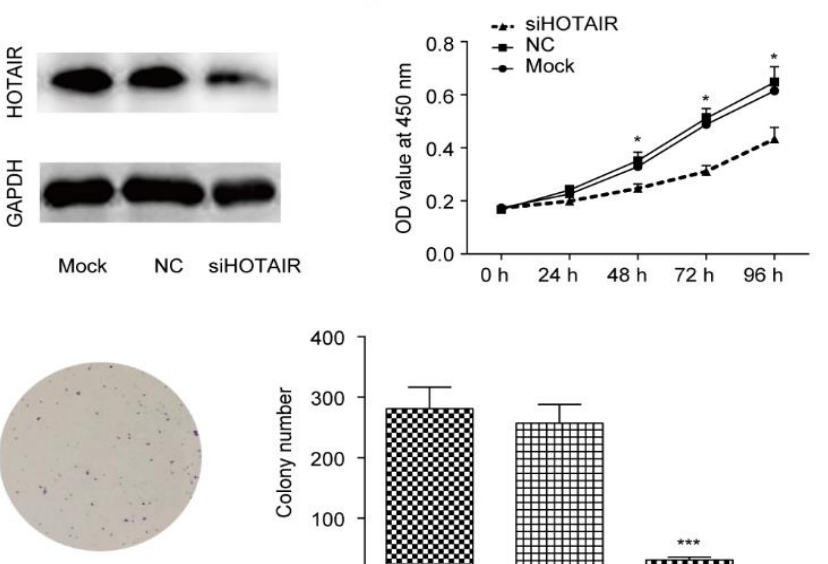

siHOTAIR
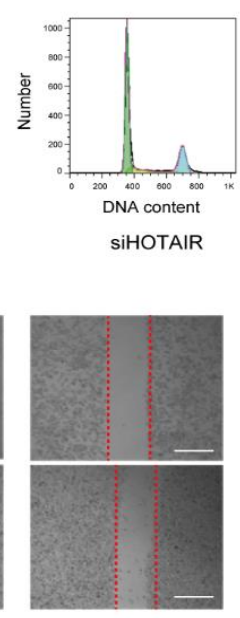

siHOTAIR
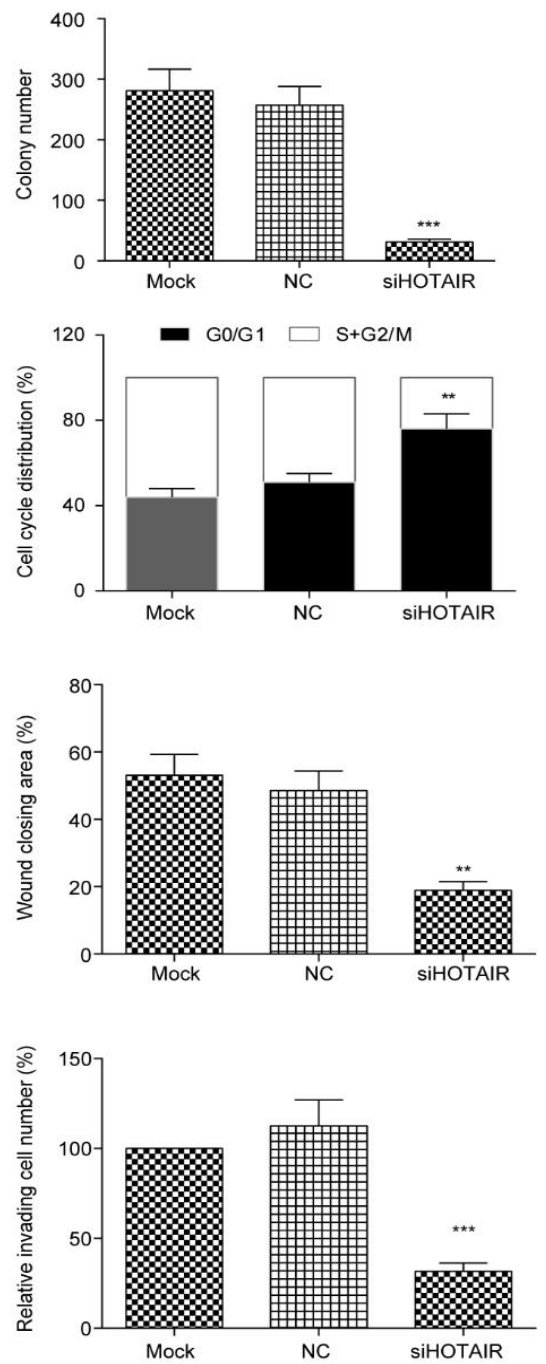

Fig. 2. Inhibition of HOTAIR reduced cell viability, proliferation, migration and invasion. (A) HOTAIR expression was lower in SKOV3 cells transfected with siHOTAIR than in cells transfected with Mock or NC. The expression of HOTAIR was normalized to GAPDH mRNA expression. ${ }^{* *} \mathrm{P}<0.001$, compared with the Mock group and the NC group. (B) The cell proliferation was lower in the siHOTAIR group than in the Mock and NC groups. ${ }^{*} \mathrm{P}<0.05$, compared with the Mock group and the NC group. (C) Colony formation ability was inhibited in SKOV3 cells transfected with siHOTAIR. ${ }^{* *} \mathrm{P}<0.001$, compared with the Mock group and the NC group. (D) Flow cytometry analysis showed that HOTAIR inhibition induced G0/G1 cell cycle arrest. ${ }^{*} \mathrm{P}<0.01$, compared with the Mock group and the NC group. (E) Cell migration ability was suppressed in SKOV3 cells transfected with siHOTAIR. " $\mathrm{P}<0.01$, compared with the Mock group and the NC group. (F) Cell invasion ability was impeded in SKOV3 cells transfected with siHOTAIR. Mock: empty vector, NC: negative control siRNA. ${ }^{* * * *} \mathrm{P}<0.001$, compared with the Mock group and the NC group. 


\section{Cellular Physiology Cell Physiol Biochem 2018;49:1289-1303 \begin{tabular}{ll|l} 
and Biochemistry $10.1159 / 000493408$ & $\begin{array}{l}\text { P } 2018 \text { The Author(s). Published by S. Karger AG, Basel } \\
\text { www.karger.com/cpb }\end{array}$
\end{tabular} \\ Chang et al.: Functions and Related Mechanism of HOTAIR in OC}

12 potential target genes of miR-206 (CCND1, CCND2, FBXW7, CDK14, CDK4, CDK9, CDK6, $P A K 3, C N N M 2, C C N C, G A K$ and NUCKS1) in 92 pairs of ovarian carcinoma and corresponding paracarcinoma tissues by qRT-PCR, as shown in Fig. 4. We found that the CCND1 and CCND2 expression levels were significantly higher in the ovarian cancer tissues than in the normal tissues (Fig. 4A-B). Therefore, we chose CCND1 and CCND2 as our genes to be further studied. Additionally, we confirmed that miR-206 directly targeted the CCND1 3' UTR and the CCND2 3' UTR (Fig. 3C-D). As shown in Fig. 3E-G, HOTAIR knockdown sharply enhanced the expression of miR-206 but repressed the expression of CCND1/CCND2 mRNA and protein. The miR-206 inhibitor had no effect on the HOTAIR expression level but markedly increased the CCND1/CCND2 mRNA and protein expression levels (Fig. 3H-J).

The correlation between CCND1/CCND2 expression and HOTAIR expression in ovarian cancer tissues

The results illustrated that high HOTAIR expression was positively correlated with histological grade, FIGO stage and metastasis (Table 1) and that high CCND2 expression was positively associated with FIGO stage in ovarian cancer. As shown in Fig. 4A and 4B, CCND1 and CCND2 were more highly expressed in the ovarian cancer tissues than in the normal tissues. The expression levels of CCND1 and CCND2 were positively correlated with the HOTAIR expression level in ovarian cancer tissues (Fig. 4M-N).

Overexpression of miR-206 inhibited the proliferation, migration and invasion of ovarian cancer cells, whereas CCND1 and CCND2 upregulation abolished the inhibition

MiR-206 overexpression in SKOV3 cells exerted a significant silencing effect on CCND1 and CCND2 expression, whereas upregulation of CCND1 or CCND2 had no obvious influence on miR-206 expression (Fig. 5A-D). As shown in Fig. 5E-F, both cell viability and colony formation capacity were decreased in SKOV3 cells after transfection with miR-206. In contrast, cell viability and colony formation ability were increased in SKOV3 cells transfected with CCND1 and CCND2 overexpression group compared with NC group. In addition, cotreatment with miR-206 and either CCND1 or CCND2 restored the miR-206 mimic-induced repression of cell proliferation and colony formation. Furthermore, the cell cycle analysis demonstrated that the overexpression of miR-206 led to cell cycle arrest at the G0/G1 phase. In contrast, CCND1 or CCND2 overexpression significantly promoted cell cycle arrest in the S and G2/M phases. Cotransfection with miR-206 and either CCND1 or CCND2 restored the miR-206 mimic-induced acceleration of G0/G1 phase transition (Fig. 5G). Moreover, as shown in Fig. 6, aberrant upregulation of miR-206 dramatically inhibited not only cell migration but also cell invasion, and these effects were neutralized by the transfection of the CCND1 or CCND2 overexpression vectors.

\section{Discussion}

In the present study, it was found that HOTAIR was overexpressed in ovarian cancer tissues and cell lines and that this overexpression promoted cancer cell activity and metastasis by targeting miR-206, therefore increasing the expression of CCND1 and CCND2. Our study not only discovered the HOTAIR/miR-206/CCND1/CCND2 regulatory network in ovarian cancer pathogenesis but also shed light on new strategies for ovarian cancer therapy.

LncRNA HOTAIR resides at the HOXC locus and has been reported to be upregulated in many types of cancers [24-29]. For instance, Xia et al. reported that HOTAIR can promote metastasis in renal cell carcinoma by upregulating the histone demethylase JMJD3 [30]. In addition, a meta-analysis highlighted that HOTAIR was overexpressed in cancer tissues and actively participated in cancer occurrence and development [31]. Consistent with these findings, our study also found that HOTAIR expression was significantly enhanced in ovarian cancer and that its depletion suppressed ovarian cancer cell aggressiveness; this result was also in agreement with recent evidence that highly expressed HOTAIR was connected with 


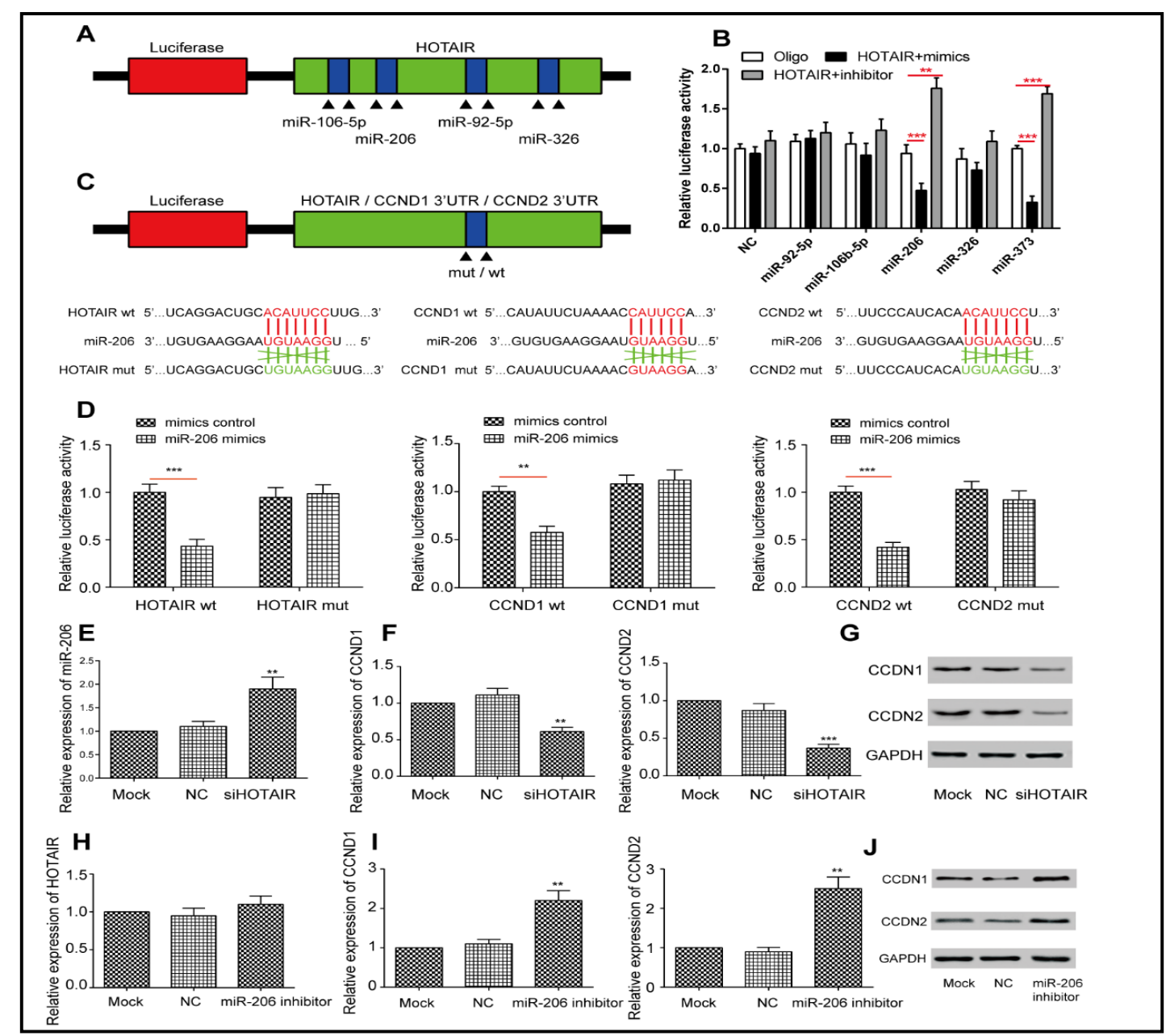

Fig. 3. HOTAIR targeted miR-206, which targeted CCND1 and CCND2. (A) The HOTAIR binding sites on miR-92-5p, miR-106b-5p, miR-206 and miR-326 are shown. (B) The luciferase reporter plasmids containing oligo control (oligo) or HOTAIR (HOTAIR) were cotransfected into SKOV3 cells along with pmirGLO plasmids containing different miRNA sequences or negative control (NC) plasmids. The transfection of the miR206 mimic significantly suppressed the luciferase activity of the HOTAIR reporters. ${ }^{* * *} \mathrm{P}<0.001$, compared with the oligo group. The miR-373 group was used as the positive control. (C) HOTAIR, CCND1 3' UTR and CCND2 3' UTR sequences were cloned into the luciferase reporter system. (D) MiR-206 mimic transfection clearly suppressed the luciferase activity in the wild-type HOTAIR, CCND1 and CCND2 groups but not in the mutated groups. ${ }^{* *} \mathrm{P}<0.01,{ }^{* * *} \mathrm{P}<0.001$, compared with the mimic control group. (E) The expression of miR206 was increased in SKOV3 cells transfected with siHOTAIR compared with that in SKOV3 cells transfected with the Mock or NC plasmids. ${ }^{* * *} \mathrm{P}<0.001$, compared with the Mock group and the NC group. The expression of miR-206 was normalized to U6 expression. (F) The mRNA levels of CCND1 and CCND2 were decreased in SKOV3 cells transfected with siHOTAIR compared with those in cells transfected with the Mock or NC plasmids. ${ }^{* *} \mathrm{P}<0.01,{ }^{* * *} \mathrm{P}<0.001$, compared with Mock group and NC group. (G) The protein expression levels of CCND1 and CCND2 were decreased in SKOV3 cells transfected with siHOTAIR compared with those in cells transfected with the Mock or NC plasmids. The expression of CCND1 or CCND2 was normalized to GAPDH expression. (H) The HOTAIR expression level in the NC, Mock and miR-206 inhibitor groups was detected by qRT-PCR. (I) The mRNA levels of CCND1 and CCND2 were increased in SKOV3 cells transfected with the miR-206 inhibitor compared with those in cells transfected with the Mock or NC plasmids. ${ }^{* *} \mathrm{P}<0.01$, compared with the Mock group and the NC group. (J) The protein expression levels of CCND1 and CCND2 were increased in SKOV3 cells transfected with the miR-206 inhibitor compared with those in cells transfected with the Mock or NC plasmids. The expression of CCND1 and CCND2 was normalized to GAPDH expression.

\section{KARGER}




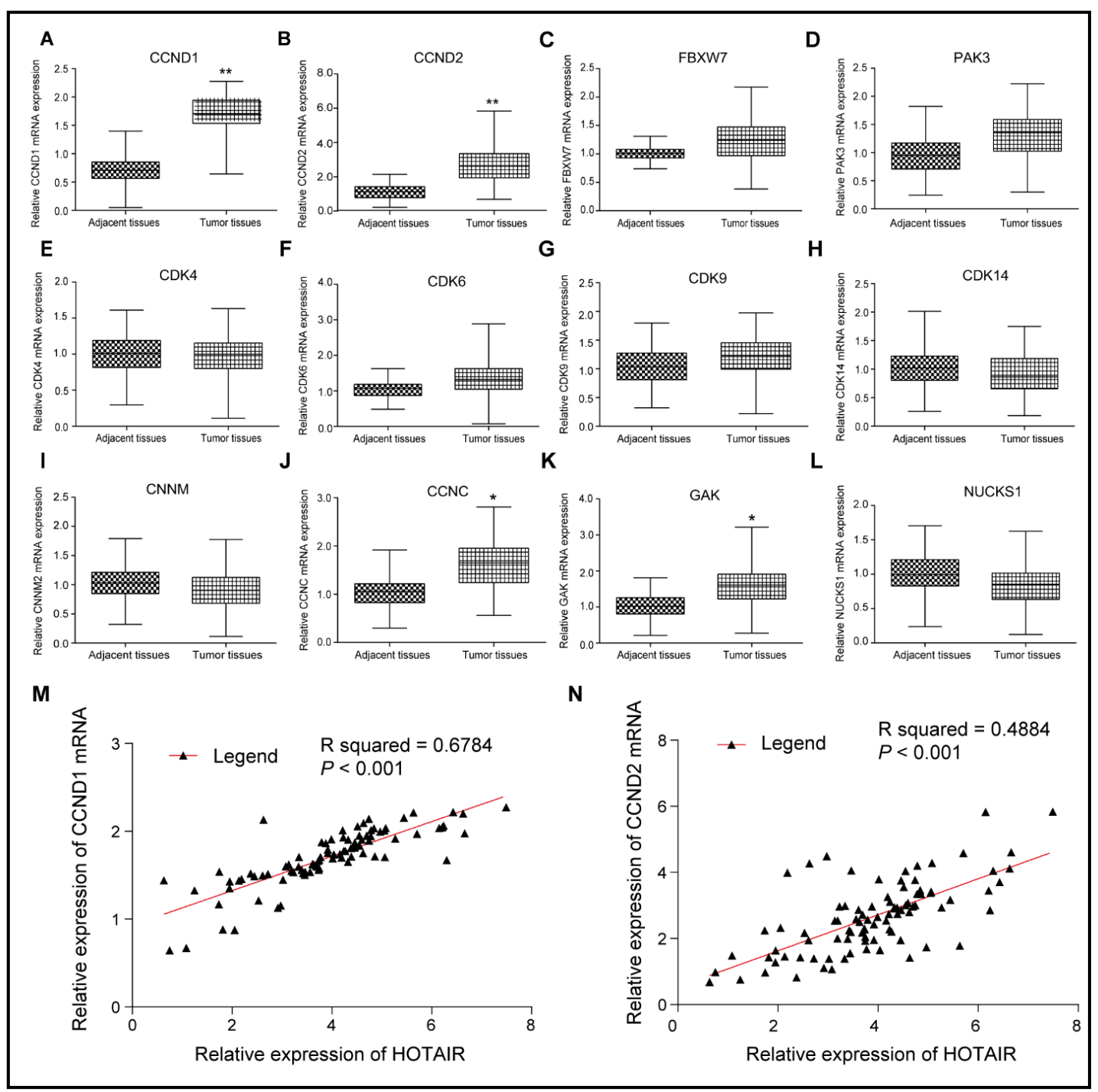

Fig. 4. The expression levels of 12 potential miR-206 target genes in 92 pairs of ovarian carcinoma and corresponding paracarcinoma tissues. (A) The expression level of CCND1 was higher in the ovarian carcinoma tissues than in the corresponding paracarcinoma tissues. ${ }^{* *} \mathrm{P}<0.01$, compared with the normal tissue group. (B) The expression level of CCND2 was higher in ovarian carcinoma tissues than in the corresponding paracarcinoma tissues. ${ }^{* *} \mathrm{P}<0.01$, compared with the normal tissue group. (C) The FBXW7 expression levels. (D) The CDK14 expression levels. (E) The CDK4 expression levels. (F) The CDK9 expression levels. (G) The CDK6 expression levels. (H) The PAK3 expression levels. (I) The CNNM2 expression levels. (J) The CCNC expression levels. ${ }^{*} \mathrm{P}<0.05$, compared with the normal tissue group. (K) The GAK expression levels. (L) The NUCKS1 expression levels. ${ }^{*} \mathrm{P}<0.05$, compared with the normal tissue group. (M) The HOTAIR expression level was positively correlated with the CCND1 level in ovarian cancer tissues $\left(\mathrm{R}^{2}=0.6784, \mathrm{P}<0.001\right)$. (N) The HOTAIR expression level was positively correlated with the CCND2 level in ovarian cancer tissues $\left(\mathrm{R}^{2}=\right.$ $0.4684, \mathrm{P}<0.001$ ). Number of tissue samples $=92$. 


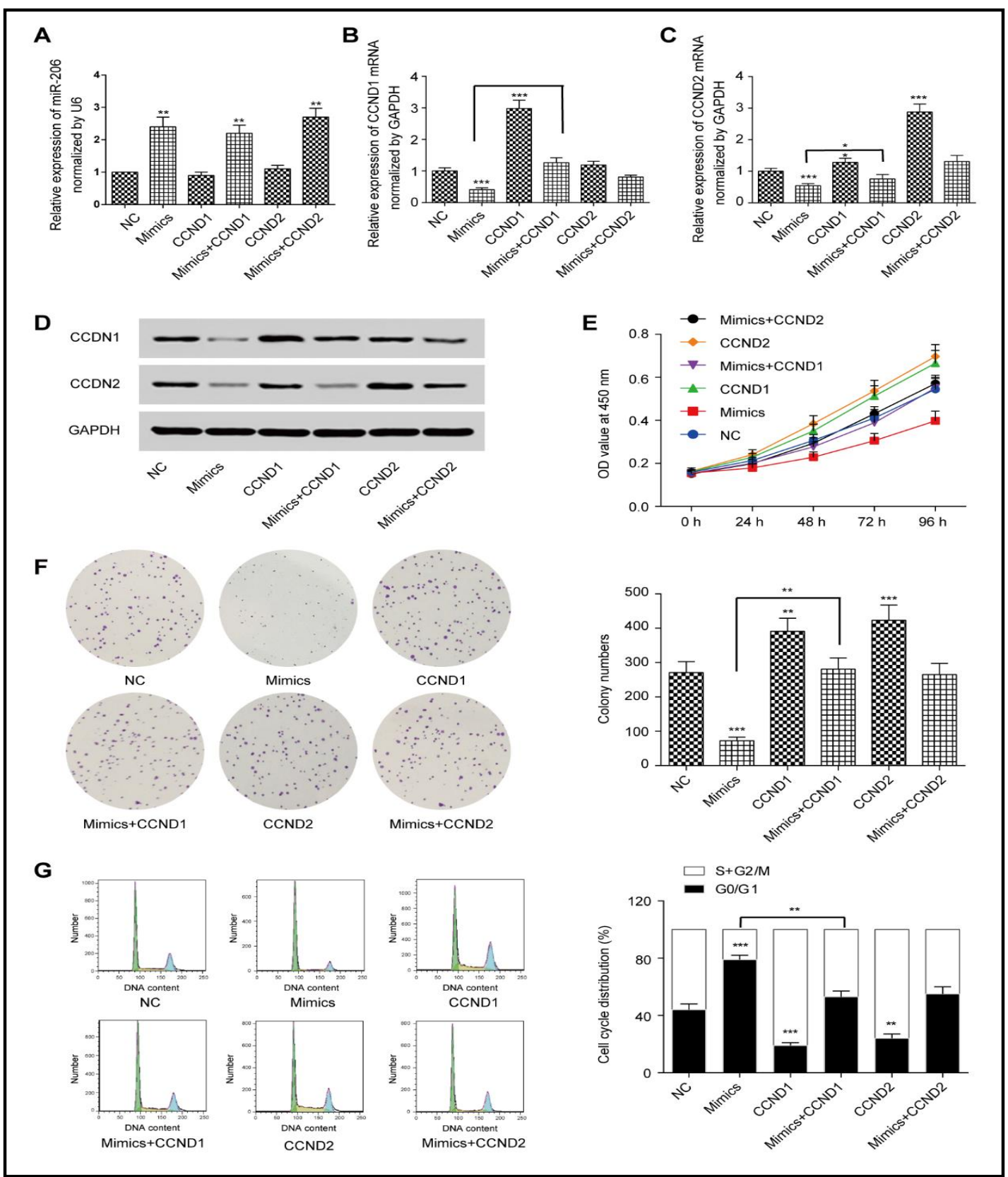

Fig. 5. Overexpression of miR-206 inhibited the proliferation of ovarian cancer cells, whereas overexpression of CCND1 and CCND2 abolished the inhibition. (A) The expression level of miR-206 was higher in SKOV3 cells transfected with Lenti-vpak packaging system-oligo + miR-206 mimic (mimic), mimic+CCND1 or mimic+CCND2 than in cells transfected with Lenti-vpak packaging system-oligo (NC), CCND1 and CCND2. (B) The expression level of CCND1 mRNA was increased in SKOV3 cells transfected with CCND1 cDNA and the promotion effect was reversed by transfection with the miR-206 mimic. (C) The relative expression level of CCND2 mRNA was increased in SKOV3 cells transfected with CCND2 cDNA and the promotion effect was reversed by transfection with the miR-206 mimic. (D) The expression of CCND1 and CCND2 in SKOV3 cells was reduced by transfection with the miR-206 mimic. (E) Cell proliferation ability was inhibited by the overexpression of miR-206, and the inhibitory effect of miR-206 was reversed by the overexpression of CCND1 and CCND2. (F) Colony formation ability was inhibited by the overexpression of miR-206, and the inhibitory effect of miR-206 was reversed by the overexpression of CCND1 and CCND2. (G) Overexpression of miR-206 induced G0/G1 cell cycle arrest, and the effect on cell cycle arrest was reversed by the overexpression of CCND1 and CCND2. ${ }^{*} \mathrm{P}<0.05,{ }^{* *} \mathrm{P}<0.01,{ }^{* * *} \mathrm{P}<0.001$, compared with the NC group. 


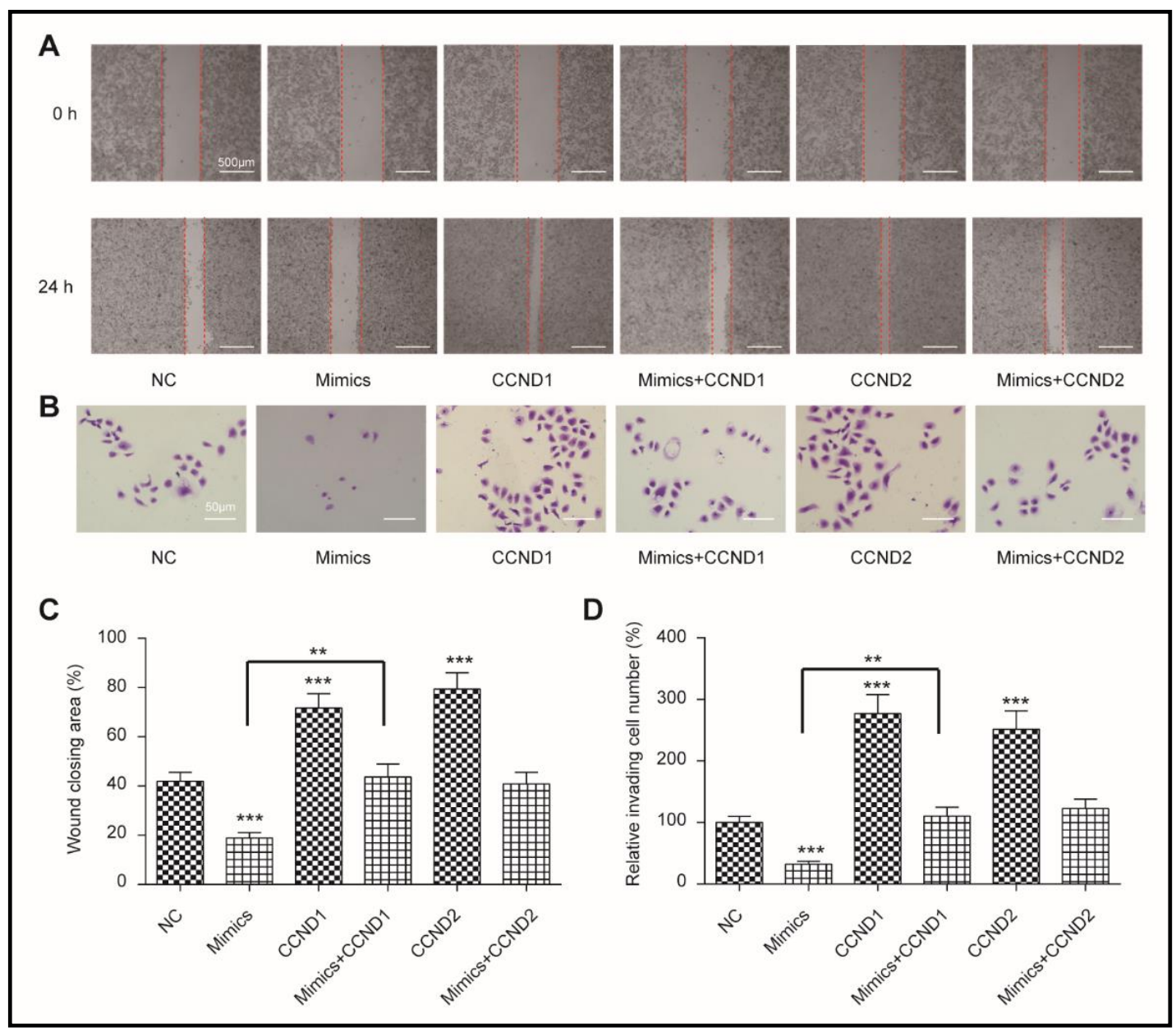

Fig. 6. Overexpression of miR-206 inhibited the migration and invasion of ovarian cancer cells, whereas overexpression of CCND1 and CCND2 abolished the inhibition. (A) Cell migration ability was inhibited by the overexpression of miR-206, and the inhibitory effect of miR-206 was reversed by the overexpression of CCND1 and CCND2. Scale bar $=500 \mu \mathrm{m}$. (B) Cell invasion ability was inhibited by the overexpression of miR-206, and the inhibitory effect of miR-206 was reversed by the overexpression of CCND1 and CCND2. Scale bar $=50 \mu \mathrm{m}$. (C) Cell migration ability was inhibited approximately 55\% by the miR-206 mimic but was enhanced by the overexpression of CCND1 or CCND2, and the inhibitory effect of miR-206 was reversed by the overexpression of CCND1 and CCND2. (D) Cell invasion ability was inhibited approximately $70 \%$ by the miR-206 mimic but was enhanced by the overexpression of CCND1 or CCND2, and the inhibitory effect of miR-206 was reversed by the overexpression of CCND1 and CCND2. ${ }^{* * *} \mathrm{P}<0.001$, compared with the NC group.

ovarian carcinoma cell proliferation and metastasis [17]. In addition, in our study, HOTAIR overexpression was found to be positively correlated with advanced histological grade, FIGO stage and extensive metastasis, indicating that HOTAIR was an excellent biomarker for the prognosis of ovarian cancer and that the downregulation of HOTAIR may arrest tumor progression and inhibit the metastatic phenotype.

Currently, the IncRNA-miRNA-mRNA regulatory network is widely recognized; in this network, lncRNA acts as a natural miRNA sponge (ceRNA) to sponge miRNAs, thus decreasing the expression of miRNA targets and affecting posttranscriptional regulation [32]. For example, it is reported that IncRNA PTENP1 functioned as a ceRNA to modulate PTEN levels by decoying miR-106b and miR-93 in gastric cancer [33]. Similarly, Wang et al. found that IncRNA TUG1 promotes migration and invasion by acting as a ceRNA of miR-335-5p in osteosarcoma cells [34]. In addition, IncRNA TUG1 has been revealed to stimulate human 
osteosarcoma tumorigenesis by sponging miR-9-5p and influencing POU2F1 expression [35]. Therefore, we hypothesized that IncRNA HOTAIR might function as a ceRNA in ovarian cancer. HOTAIR was directly associated with the putative miR-206 miRNA response element, thereby downregulating CCND1 or CCND2 expression. The above results suggested that HOTAIR positively modulated the expression of CCND1 and CCND2 through suppressing miR-206.

CCND1 and CCND2 belong to the D-type cyclin family, which is commonly regarded as a human cancer promoter. The elevation of CCND1 and CCND2 expression was associated with cancer metastasis $[18,19,36]$. The research performed by the Delmer group demonstrated that CCND2 was overexpressed in chronic B cell malignancies [37]. Additionally, a metaanalysis of observational studies verified that CCND1 was upregulated in colorectal cancer and was associated with poor clinical outcome, TNM staging and metastasis [38]. Similar to previous studies, our study also discovered that the levels of both CCND1 and CCND2 were high and were positively correlated with the HOTAIR level in ovarian cancer. Thus, we speculated that the downregulation of CCND1/CCND2 might be a novel ovarian cancer therapy target, and we did find that CCND1/CCND2 inhibition resulted in decreased cancer cell proliferation as well as migration, whereas the overexpression of CCND1/CCND2 resulted in enhanced proliferation and metastasis.

However, limitations still exist in this study. First, it is certain that other lncRNAs exist that serve as ceRNAs to regulate the expression of crucial genes in ovarian cancer progression. Second, HOTAIR can simultaneously bind to a large number of miRNAs, some of which can also affect the development of ovarian cancer. Indeed, neutralization of miR-206 did not positively affect the levels of HOTAIR expression but did increase the levels of CCND1 and CCND2 expression. Additionally, in vivo experiments are required in further studies to confirm the role of the HOTAIR-miR-206-CCND1/CCND2 network in ovarian cancer.

In the study, HOTAIR positively stimulated CCND1 and CCND2 expression by negatively modulating miR-206 expression through a ceRNA regulatory network, thereby promoting ovarian cancer cell proliferation, cell cycle progression, migration and invasion.

\section{Conclusion}

In summary, this study highlighted that HOTAIR, as an oncogene, exerted a crucial role in the proliferation, metastasis and invasion of ovarian cancer cells. In addition, a novel HOTAIR-miR-206-CCND1/CCND2 pathway regulatory axis, in which HOTAIR enhanced CCND1 and CCND2 expression by directly suppressing miR-206, was revealed to be involved in promoting ovarian tumorigenesis. Finally, our study suggested that targeting the HOTAIRmiR-206-CCND1/CCND2 pathway might present a novel therapeutic viewpoint and offer a better understanding of the pathogenesis and development of ovarian cancer.

\section{Acknowledgements}

This work was funded by China Postdoctoral Science Foundation (2018T110743) and Technology Foundation for Selected Overseas Chinese Scholar.

\section{Disclosure Statement}

The authors declare to have no competing interests. 


\section{Cellular Physiology Cell Physiol Biochem 2018;49:1289-1303 \begin{tabular}{l|l|l} 
and Biochemistry & Dublished online: 12 September, 2018 & $\begin{array}{l}\text { (c) } 2018 \text { The Author(s). Published by S. Karger AG, Basel } \\
\text { www.karger.com/cpb }\end{array}$
\end{tabular}}

\section{References}

1 Siegel RL, Miller KD, Jemal A: Cancer statistics, 2016. CA Cancer J Clin 2016;66:7-30.

- Bast RC, Jr., Hennessy B, Mills GB: The biology of ovarian cancer: new opportunities for translation. Nat Rev Cancer 2009;9:415-428.

3 Holmes D: Ovarian cancer: beyond resistance. Nature 2015;527:S217.

-4 Brinkhuis M, Izquierdo MA, Baak JP, van Diest PJ, Kenemans P, Scheffer GL, Scheper RJ: Expression of multidrug resistance-associated markers, their relation to quantitative pathologic tumour characteristics and prognosis in advanced ovarian cancer. Anal Cell Pathol 2002;24:17-23.

-5 Xue M, Zhuo Y, Shan B: MicroRNAs, Long Noncoding RNAs, and Their Functions in Human Disease. Methods Mol Biol 2017;1617:1-25.

-6 Yoshida N, Kimura T: Pathogen-associated regulatory non-coding RNAs and oncogenesis. Front Biosci (Landmark Ed) 2017;22:1599-1621. Farazi TA, Hoell JI, Morozov P, Tuschl T: MicroRNAs in human cancer. Adv Exp Med Biol 2013;774:1-20. Bartel DP: MicroRNAs: genomics, biogenesis, mechanism, and function. Cell 2004;116:281-297. Bhayani MK, Calin GA, Lai SY: Functional relevance of miRNA sequences in human disease. Mutat Res 2012;731:14-19.

10 Salmena L, Poliseno L, Tay Y, Kats L, Pandolfi PP: A ceRNA hypothesis: the Rosetta Stone of a hidden RNA language? Cell 2011;146:353-358.

11 Li H, Tang XM, Liu Y, Li W, Chen Q, Pan Y: Association of Functional Genetic Variants of HOTAIR with Hepatocellular Carcinoma (HCC) Susceptibility in a Chinese Population. Cell Physiol Biochem 2017;44:447454.

12 Gupta RA, Shah N, Wang KC, Kim J, Horlings HM, Wong DJ, Tsai MC, Hung T, Argani P, Rinn JL, Wang Y, Brzoska P, Kong B, Li R, West RB, van de Vijver MJ, Sukumar S, Chang HY: Long non-coding RNA HOTAIR reprograms chromatin state to promote cancer metastasis. Nature 2010;464:1071-1076.

13 Wang L, Yang F, Jia LT, Yang AG: Missing Links in Epithelial-Mesenchymal Transition: Long Non-Coding RNAs Enter the Arena. Cell Physiol Biochem 2017;44:1665-1680.

14 Lai Y, He S, Ma L, Lin H, Ren B, Ma J, Zhu X, Zhuang S: HOTAIR functions as a competing endogenous RNA to regulate PTEN expression by inhibiting miR-19 in cardiac hypertrophy. Mol Cell Biochem 2017;432:179187.

15 Kogo R, Shimamura T, Mimori K, Kawahara K, Imoto S, Sudo T, Tanaka F, Shibata K, Suzuki A, Komune S, Miyano S, Mori M: Long noncoding RNA HOTAIR regulates polycomb-dependent chromatin modification and is associated with poor prognosis in colorectal cancers. Cancer Res 2011;71:6320-6326.

-16 Rinn JL, Kertesz M, Wang JK, Squazzo SL, Xu X, Brugmann SA, Goodnough LH, Helms JA, Farnham PJ, Segal E, Chang HY: Functional demarcation of active and silent chromatin domains in human HOX loci by noncoding RNAs. Cell 2007;129:1311-1323.

17 Zhang Z, Cheng J, Wu Y, Qiu J, Sun Y, Tong X: LncRNA HOTAIR controls the expression of Rab22a by sponging miR-373 in ovarian cancer. Mol Med Rep 2016;14:2465-2472.

-18 Ahlin C, Lundgren C, Embretsen-Varro E, Jirstrom K, Blomqvist C, Fjallskog M: High expression of cyclin D1 is associated to high proliferation rate and increased risk of mortality in women with ER-positive but not in ER-negative breast cancers. Breast Cancer Res Treat 2017;164:667-678.

19 Lee E, Jin D, Lee BB, Kim Y, Han J, Shim YM, Kim DH: Negative effect of cyclin D1 overexpression on recurrence-free survival in stage II-IIIA lung adenocarcinoma and its expression modulation by vorinostat in vitro. BMC Cancer 2015;15:982.

20 Zhou JJ, Cheng D, He XY, Meng Z, Li WZ, Chen RF: Knockdown of Hotair suppresses proliferation and cell cycle progression in hepatocellular carcinoma cell by downregulating CCND1 expression. Mol Med Rep 2017;16:4980-4986.

21 Di W, Li Q Shen W, Guo H, Zhao S: The long non-coding RNA HOTAIR promotes thyroid cancer cell growth, invasion and migration through the miR-1-CCND2 axis. Am J Cancer Res 2017;7:1298-1309.

22 Dai J, Wei RJ, Li R, Feng JB, Yu YL, Liu PS: A study of CCND1 with epithelial ovarian cancer cell proliferation and apoptosis. Eur Rev Med Pharmacol Sci 2016;20:4230-4235.

-23 Kolijn K, van Leenders GJ: Comparison of RNA extraction kits and histological stains for laser capture 


\section{Cellular Physiology Cell Physiol Biochem 2018;49:1289-1303 \begin{tabular}{l|l} 
DOI: 10.1159/000493408 & $\begin{array}{l}\text { O 2018 The Author(s). Published by S. Karger AG, Basel } \\
\text { www.karger.com/cpb }\end{array}$
\end{tabular} \\ Chang et al.: Functions and Related Mechanism of HOTAIR in OC}

microdissected prostate tissue. BMC Res Notes 2016;9:17.

24 Cai H, Yao J, An Y, Chen X, Chen W, Wu D, Luo B, Yang Y, Jiang Y, Sun D, He X: LncRNA HOTAIR acts a competing endogenous RNA to control the expression of notch3 via sponging miR-613 in pancreatic cancer. Oncotarget 2017;8:32905-32917.

25 Ren K, Li Y, Lu H, Li Z, Li Z, Wu K, Li Z, Han X: Long Noncoding RNA HOTAIR Controls Cell Cycle by Functioning as a Competing Endogenous RNA in Esophageal Squamous Cell Carcinoma. Transl Oncol 2016;9:489-497.

-26 Worku T, Bhattarai D, Ayers D, Wang K, Wang C, Rehman ZU, Talpur HS, Yang L: Long Non-Coding RNAs: the New Horizon of Gene Regulation in Ovarian Cancer. Cell Physiol Biochem 2017;44:948-966.

27 Wu Y, Zhang L, Wang Y, Li H, Ren X, Wei F, Yu W, Wang X, Zhang L, Yu J, Hao X: Long noncoding RNA HOTAIR involvement in cancer. Tumour Biol 2014;35:9531-9538.

28 Xu CZ, Jiang C, Wu Q Liu L, Yan X, Shi R: A Feed-Forward Regulatory Loop between HuR and the Long Noncoding RNA HOTAIR Promotes Head and Neck Squamous Cell Carcinoma Progression and Metastasis. Cell Physiol Biochem 2016;40:1039-1051.

29 Yu D, Zhang C, Gui J: RNA-binding protein HuR promotes bladder cancer progression by competitively binding to the long noncoding HOTAIR with miR-1. Onco Targets Ther 2017;10:2609-2619.

30 Xia M, Yao L, Zhang Q, Wang F, Mei H, Guo X, Huang W: Long noncoding RNA HOTAIR promotes metastasis of renal cell carcinoma by up-regulating histone H3K27 demethylase JMJD3. Oncotarget 2017;8:1979519802.

-31 Hu C, Yu D, Sun X, Zhang M, Wang L, Qin H: The prevalence and progression of mild cognitive impairment among clinic and community populations: a systematic review and meta-analysis. Int Psychogeriatr 2017;29:1595-1608.

-32 Liu D, Yu X, Wang S, Dai E, Jiang L, Wang J, Yang Q, Yang F, Zhou S, Jiang W: The gain and loss of long noncoding RNA associated-competing endogenous RNAs in prostate cancer. Oncotarget 2016;7:5722857238.

-33 Zhang R, Guo Y, Ma Z, Ma G, Xue Q Li F, Liu L: Long non-coding RNA PTENP1 functions as a ceRNA to modulate PTEN level by decoying miR-106b and miR-93 in gastric cancer. Oncotarget 2017;8:2607926089.

-34 Wang Y, Yang T, Zhang Z, Lu M, Zhao W, Zeng X, Zhang W: Long non-coding RNA TUG1 promotes migration and invasion by acting as a ceRNA of miR-335-5p in osteosarcoma cells. Cancer Sci 2017;108:859-867.

-35 Xie CH, Cao YM, Huang Y, Shi QW, Guo JH, Fan ZW, Li JG, Chen BW, Wu BY: Long non-coding RNA TUG1 contributes to tumorigenesis of human osteosarcoma by sponging miR-9-5p and regulating POU2F1 expression. Tumour Biol 2016;37:15031-15041.

-36 Takano Y, Kato Y, Masuda M, Ohshima Y, Okayasu I: Cyclin D2, but not cyclin D1, overexpression closely correlates with gastric cancer progression and prognosis. J Pathol 1999;189:194-200.

-37 Delmer A, Ajchenbaum-Cymbalista F, Tang R, Ramond S, Faussat AM, Marie JP, Zittoun R: Overexpression of cyclin D2 in chronic B-cell malignancies. Blood 1995;85:2870-2876.

-38 Li Y, Wei J, Xu C, Zhao Z, You T: Prognostic significance of cyclin D1 expression in colorectal cancer: a metaanalysis of observational studies. PLoS One 2014;9:e94508. 